\title{
SPOTS OVER ANALYTICALLY NORMAL LOCAL DOMAINS
}

\author{
JUDITH D. SALLY
}

(Communicated by Louis J. Ratliff, Jr.)

\begin{abstract}
A result of Lipman on 2-dimensional analytically normal local domains is extended to dimension $d>2$, thereby enlarging the class of local domains known to have certain finiteness properties with respect to birational extensions.
\end{abstract}

This note is an addendum to the paper, $A$ criterion for spots [HHS]. Its purpose is to extend a result of Lipman [L] to dimension $d>2$, and thereby enlarge the class of $d$-dimensional local domains known to have the property that every $d$ dimensional quasi-unmixed local domain birationally dominating them is a spot over them. (Recall that a local ring $S$ is said to be a spot over a ring $R$ if $S$ is the localization at a prime ideal of a finitely generated ring over $R$.)

In [HHS] the following property is defined.

DEFINITION. A $d$-dimensional local domain $(R, m)$ has property $L$, respectively $\bar{L}$, if every $d$-dimensional normal spot birationally dominating $R$ is analytically irreducible, respectively analytically normal. In [HHS] it is proved that if $(R, m)$ is a $d$-dimensional analytically unramified local domain with property $L$, then every $d$-dimensional normal or quasi-unmixed local domain which birationally dominates $R$ is a spot over $R$.

The class of local domains with $\bar{L}$ includes all one dimensional local domains, all excellent local domains and, by a result of Lipman $[\mathbf{L}]$, all 2-dimensional analytically normal domains. It is an open question whether all $d$-dimensional analytically normal local domains, for $d>2$, are in this class. The following result extends Lipman's result to certain $d$-dimensional analytically normal local domains.

PROPOSITION. Let $(R, m)$ be a d-dimensional analytically normal local domain such that $R / p$ is Nagata for all height 2 primes $p$ of $R$. Then $R$ has $\bar{L}$. In fact, every normal spot which birationally dominates $R$ is analytically normal.

ProOF. Let $(S, n)$ be a normal local domain birationally dominating $R$ which is a spot over $R$. We will show that $S$ is analytically normal. With - denoting maximal ideal-adic completion, we have

$$
R \subset \hat{R} \subset \hat{R} \otimes_{R} S \subset\left(\hat{R} \otimes_{R} S\right)_{n\left(\hat{R} \otimes_{R} S\right)}=T .
$$

Now

$$
\hat{R} \otimes_{R} S / n^{i}\left(\hat{R} \otimes_{R} S\right) \cong \hat{R} \otimes_{R}\left(S / n^{i} S\right) \cong R / m^{i} R \otimes_{R / m^{i} R} S / n^{i} S \cong S / n^{i} S,
$$

Received by the editors April 24, 1987.

1980 Mathematics Subject Classification (1985 Revision). Primary 13E15, 13B30; Secondary 13B35, $13 \mathrm{C} 15$.

The author is partially supported by a grant from the National Science Foundation. 
so $\hat{T}=\hat{S}$. Since $T$ is a spot over $\hat{R}, T$ is excellent. Thus to prove that $S$ is analytically normal it is sufficient to prove that $T$ is normal.

$T$ is flat over $S$, so for any prime ideal $P$ of $T$

$$
\operatorname{depth} T_{P}=\operatorname{depth} S_{Q}+\operatorname{depth} T_{P} / Q T_{P}
$$

where $Q=P \cap S$. We will prove that $T_{P}$ is normal for all primes $P$ of $T$ which are maximal primes of principal ideals of $T$ [K, Theorem 53]. Let $P$ be one such. Let $p=P \cap R$ and $Q=P \cap S$. Suppose first that ht $p \leq 1$. Then $R_{p}=S_{Q}$ and $T_{P}$ is a localization of $\hat{R} \otimes_{R} R_{p} \cong \hat{R}_{R-p}$ which is normal since $\hat{R}$ is normal.

Suppose now that ht $p>1$. Since $P$ is a maximal prime of a principal ideal of $T$, depth $T_{P}=1$. It follows from (*) that ht $Q=1$ because $S$ is normal. Now $R / p \subset S / Q$ so $S / Q$ is a domain which is a spot over the Nagata domain $R / p$. Consequently, $S / Q$ is analytically unramified, i.e., $Q \hat{S}$ is a radical idea. Since $P \supseteq Q T=Q \hat{T} \cap T=Q \hat{S} \cap T$, it follows that $T / Q T \subset \hat{S} / Q \hat{S}$. Hence $Q T$ is a radical ideal and $T_{P} / Q T_{P}$ is reduced. Now $S_{Q}$ is a DVR so $Q S_{Q}=x S_{Q}$, for some $x \in Q$. Thus $P T_{P}=Q T_{P}=\left(Q S_{Q}\right) T_{P}=x T_{P}$, and $T_{P}$ is also a DVR.

\section{REFERENCES}

[HHS] W. Heinzer, C. Huneke and J. D. Sally, A criterion for spots, Kyoto J. Math. 26 (1986).

[K] I. Kaplansky, Commutative rings, Allyn and Bacon, Boston, Mass., 1970.

[L] J. Lipman, Desingularization of two-dimensional schemes, Ann. of Math. (2) 107 (1978), 151207. 60201

DEPARTMENT OF MATHEMATICS, NORThWESTERN UNIVERSity, EVANSTON, ILlinOIS 